\title{
Current antibiotic resistance patterns of rare uropathogens: survey from Central European Urology Department 2011-2019
}

\author{
Jan Hrbacek ${ }^{1 *} \mathbb{D}$, Pavel Cermak ${ }^{2}$ and Roman Zachoval ${ }^{1}$
}

\begin{abstract}
Background: While the resistance rates of commonly detected uropathogens are well described, those of less frequent Gram-negative uropathogenic bacteria have seldom been reported. The aim of this study was to examine the resistance rates of less frequent uropathogenic Gram-negatives in a population of patients treated in a Department of Urology of a tertiary referral centre in Central Europe over a period of 9 years.

Methods: Data on all positive urine samples from urological in- and out-patients were extracted form the Department of Clinical Microbiology database from 2011 to 2019. Numbers of susceptible and resistant isolates per year were calculated for these uropathogens: Acinetobacter spp. $(n=74)$, Citrobacter spp. $(n=60)$, Enterobacter spp. $(n=250)$, Morganella morganii $(n=194)$, Providencia spp. $(n=53)$, Serratia spp. $(n=82)$ and Stenotrophomonas maltophilia $(n=27)$. Antimicrobial agents selected for the survey included: ampicillin, amoxicillin/clavulanic acid, piperacillin/tazobactam; cefuroxime, cefotaxime, ceftazidime and cefepime; ciprofloxacin and ofloxacin; gentamicin and amikacin; ertapenem, meropenem and imipenem; trimethoprim-sulfamethoxazole (co-trimoxazole), nitrofurantoin and colistin.
\end{abstract}

Results: Penicillin derivatives have generally poor effect except piperacillin/tazobactam. Cefuroxime is not efficient unlike cefotaxime (except against Acinetobacter spp. and S. maltophilia). Susceptibility to fluoroquinolones is limited. Amikacin is somewhat more efficient than gentamicine but susceptibilities for both safely exceed $80 \%$. Nitrofurantoin shows virtually no efficiency. Cotrimoxazole acts well against Citrobacter spp., Serratia spp. and it is the treatment of choice for S. maltophilia UTIs. Among carbapenems, ertapenem was less efficient than meropenem and imipenem except for S. maltophilia whose isolates were mostly not suceptible to any carbapenems.

Conclusions: Uropathogenic microorganisms covered in this report are noteworthy for their frequently multi-drug resistant phenotypes. Knowledge of resistance patterns helps clinicians choose the right empirical antibiotic treatment when the taxonomical assignment of the isolate is known but sensitivity results are pending.

Keywords: Urinary tract infections, Drug resistance, Bacterial, Anti-infective agents, Urinary, Acinetobacter, Citrobacter, Enterobacter, Morganella, Providencia, Serratia, Stenotrophomonas

*Correspondence: jan.hrbacek@ftn.cz

1 Department of Urology, 3rd Faculty of Medicine, Charles University and Thomayer University Hospital, Videnska 800, 14059 Prague, Czech Republic

Full list of author information is available at the end of the article

\section{Background}

With approximately $150-250$ million cases occurring globally per year $[1,2]$, urinary tract infections (UTIs) represent some of the most common infectious diseases in humans. Gram-negative enteric bacteria such as Escherichia coli, Klebsiella spp. and Proteus spp. are 
their most common causative agents. UTIs occur more frequently among women than in men: $50 \%$ of females will experience at least one UTI in their lifetime [3]. Recurrences are common and besides associated morbidity, UTIs put pressure on health care systems, too. While the cost of treatment of a single UTI episode is insignificant, their frequent incidence means they consume a non-negligible part of the health care budget: in France alone, the expense for adult female UTIs amounted to 58 million euro in 2012 [4]. Complicated UTIs are even more costly: one episode of a complicated UTI costs between 4028 and 7740 euro [5] depending on the health care system.

Antimicrobial resistance (AMR) has become a major concern threathening many an advance of medicine in the twenty-first century. Uropathogenic microorganisms are no exception to this: rates of AMR among Enterobacteriaceae are increasing globally, albeit with temporal and spatial differences [6-15]. Antibiotic consumption is a primary driver for the spread of AMR as evidenced on local and country levels [16, 17]. For instance, southern European states exhibit higher AMR rates than northern ones. Of note, four top antibiotic prescribers in Europe (Greece, Cyprus, France and Italy) belong among south European countries [18].

International guidelines for UTI treatment are freely available; yet, poor antibiotic prescription remains common. Thirty percent of primary care antibiotic prescriptions were inadequate in a report from the United States [19]. Appropriate antibiotic treatment was reported in $68 \%$ of adult cystitis cases and only $46 \%$ of pyelonephritis cases in another survey [20].

One of the ways to improve the quality of antibiotic prescribing is antibiotic stewardship including the monitoring of local antimicrobial susceptibility patterns [6]. While AMR patterns of the commonest causative uropathogenic agents-E. coli, Klebsiella spp., Proteus spp., Pseudomonas aeruginosa etc. have been widely reported, less attention has been devoted to microbes at the bottom of the prevalence ladder. These microorganisms constitute only a small fraction of UTI etiological agents but frequently display a multi-drug resistant phenotype, presenting a therapeutic challenge [21, 22]. Furthermore, they often have the ability to survive in hostile environments such as dry surfaces, nutrient-poor aqueous solutions [23, 24] and require a particularly attentive nursing care.

The aim of this retrospective observational study was to report the AMR patterns for seven less frequent causative agents of UTIs in a Central European tertiary referral centre Department of Urology over a period of nine years.

\section{Methods}

Similar to our previous related work [25], urinary cultures from the Department of Clinical Microbiology electronic database between January 2011 and December 2019 were searched for those caused by the following genera: Acinetobacter, Citrobacter, Enterobacter, Morganella, Providencia, Serratia and Stenotrophomonas. Urine cultures may have originated from spontaneously voided midstream samples, aseptic catheterisation during theatre procedures, indwelling catheters, suprapubic catheters, nephrostomy tubes and uretero-ileostomies. Because of the small prevalence of these uropathogens, inpatient and outpatient samples were included in the analysis. Duplicates were eliminated, allowing only one isolate of a given pathogen per patient per year. Prevalence of uropathogenic organisms and their antimicrobial susceptibility patterns (including trends where appropriate) were analysed.

\section{Culture methods and susceptibility testing}

The method used for culture and susceptibility testing has been described elsewhere [25]. Briefly, uncentrifuged urine was inoculated with a $0.01 \mathrm{ml}$ loop on blood and UriSelect chromogenic agar (Bio-Rad, Berkeley, CA, USA) in a semi-quantitative dilution method. Urine diluted in saline $(1: 10)$ was inoculated with a $0.01 \mathrm{~mL}$ and $0.001 \mathrm{~mL}$ loop on Columbia blood agar (Bio-Rad, Berkeley, CA, USA) and UriSelect chromogenic agar. Agar plates were incubated at $37{ }^{\circ} \mathrm{C}$ for 20-24 h. Microorganisms were identified by their phenotypical characteristic and using the semi-automatic system MIKROLATEST ID (Erba-Lachema, Brno, Czech Republic).

Antibiotic susceptibility testing was performed by the disc diffusion method on Mueller-Hinton agar (Bio-Rad, Berkeley, CA, USA) and by the MIC dilution method (TRIOS MIC, Prague, Czech Republic until 2017, then MICRO-LA-TEST ATB (MIC) Erba-Lachema, Brno, Czech Republic). EUCAST MIC breakpoint tables were used. Intermediate results were excluded from this analysis. The cut-off used for significant bacterial presence was $10^{5}$ colony-forming units $/ \mathrm{mL}\left(10^{3}\right.$ for aseptically catheterised urine specimens).

This survey covers the following antimicrobial agents: ampicillin, amoxicillin/clavulanate and piperacillin/ tazobactam; cefuroxime, cefotaxime, ceftazidime and cefepime; ciprofloxacin and ofloxacin; gentamicin and amikacin; ertapenem, meropenem and imipenem; trimethoprim-sulfamethoxazole (co-trimoxazole), nitrofurantoin and colistin.

Cochrane-Armitage test was used to assess statistical significance of trends. Statistical analyses were 
performed in XLSTAT 2020.1.3 (Addinsoft, New York, USA). Alpha level of 0.05 was considered significant.

\section{Results}

From a total of 15,909 positive urine cultures (duplicates excluded) between 1 January 2011 and 31 December 2019, the pathogens surveyed in the present study were detected in 740 samples (4.65\%, range 3.33-6.03\% of all positive urine cultures per year, no evidence for trend in incidence rate $[\mathrm{p}=0.23]$ ) from 607 patients. Of these, 466 (76.8\%) were males and 141 (23.2\%) were females. Mean age (interquartile range) was 70.3 (64-77) and 69.2 (59-76) for men and women, respectively. Two-thirds of urine samples $(n=482)$ originated from out-patients and 258 from in-patients (see Additional file 2: Table S7 for details). Enterobacter spp. was the most prevalent uropathogen during the study period ( $\mathrm{n}=250,33.8 \%$ ) followed by Morganella morganii ( $\mathrm{n}=194,26.2 \%)$. The isolates identified included Acinetobacter spp. $(\mathrm{n}=74)$, Citrobacter spp. $(\mathrm{n}=60$, including C. braakii $(\mathrm{n}=1), C$. freundii $(\mathrm{n}=2), C$. koseri $(\mathrm{n}=23))$, Enterobacter spp. $(\mathrm{n}=250$, including E. aerogenes $(\mathrm{n}=3)$, E. cloacae $(\mathrm{n}=12))$, Morganella morganii $(\mathrm{n}=194)$, Providencia spp. $(\mathrm{n}=53$, including $P$. alcalifaciens $(\mathrm{n}=2), P$. rettgeri $(\mathrm{n}=11), P$. stuartii $(\mathrm{n}=32))$, Serratia spp. $(\mathrm{n}=82$, including $S$. ficaria $(\mathrm{n}=4)$, S. marcescens $(\mathrm{n}=10)$, S. odorifera $(\mathrm{n}=1))$ and Stenotrophomonas maltophilia $(\mathrm{n}=27)$.

There was no trend in the yearly prevalence of individual uropathogens (Fig. 1). Trends in resistance rates were computed for Enterobacter spp. and M. morganii where the number of isolates was sufficient to justify such analysis (Additional file 1: Table S1-S6).

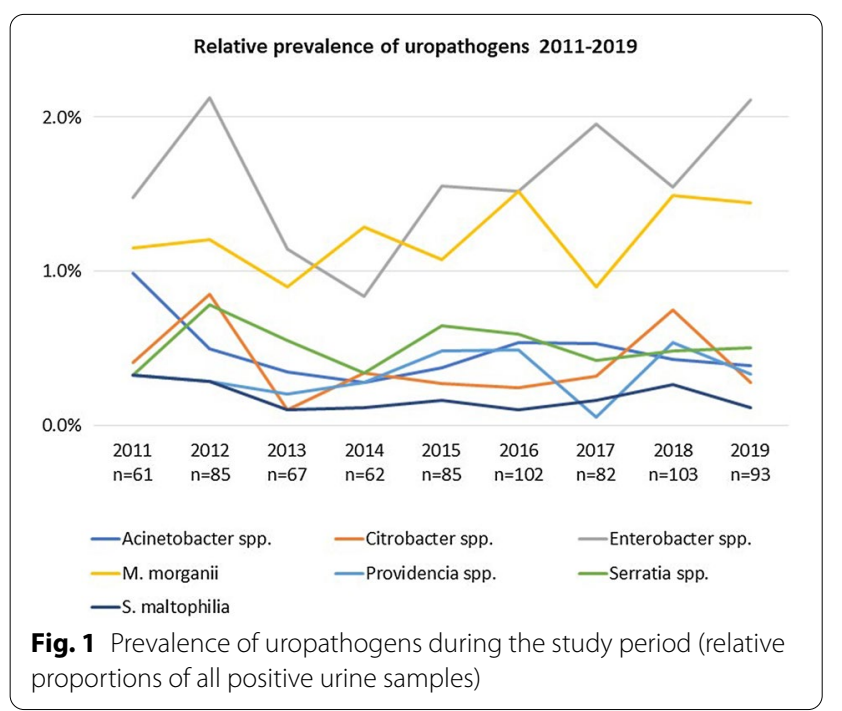

\section{Antimicrobial resistance Penicillin derivatives}

Cumulative resistance rates calculated for the entire study period exceeded $80 \%$ for ampicillin and amoxicillin/clavulanic acid for all uropathogens. Piperacillin/ tazobactam resistance did not exceed 10\% in Citrobacter spp. and Providentia spp.; it was $16.7 \%, 15.4 \%$ and 13.6\% for Acinetobacter spp., Serratia spp. and M. morganii, respectively. Table 1 summarizes resistance rates for the entire study period. Additional file 1: Table S1 gives details on the yearly number of isolates over the study period and their resistance rates to penicillins.

\section{Cephalosporines}

All uropathogens were resistant $(>50 \%)$ to secondgeneration cephalosporine, cefuroxime. Citrobacter spp. susceptibility to 3rd generation cephalosporines, cefotaxime and ceftazidime approximated 5\%. Serratia spp. was reasonably susceptible to ceftazidime and cefepime. Providentia spp. retained good susceptibility to cefepime. See Additional file 1: Table S2, for details on cephalosporine resistance rates.

\section{Fluoroquinolones}

Resistance rates of all uropathogens to ciprofloxacin exceeded $20 \%$ and approximated $60 \%$ in the case of $S$. maltophilia. Resistance rates to ofloxacin were somewhat lower but never less than 15\%. Additional file 1: Table S3 presents details on fluoroquinolone resistance rates.

\section{Aminoglycosides}

Citrobacter spp. and Serratia spp. resistance to gentamicin was just above $6 \%$. Resistance rates of the other uropathogens to gentamicin varied between $12.4 \%$ and 33.3\%. All but Acinetobacter spp. and S. maltophilia retained good susceptibility to amikacin (see Additional file 1: Table S4 for details).

\section{Carbapenems}

While Citrobacter spp. and Providentia spp. retained good suceptibility $(<10 \%)$ to all carbapenems, resistance rates to ertapenem exceeded $10 \%$ in all other bacteria examined. S. maltophilia resistance rate to all three carbapenems was above $60 \%$; Additional file 1: Table S5 gives a detailed overview.

\section{Colistin, nitrofurantoin and cotrimoxazole}

Colistin had poor effect on the pathogens surveyed in this report and their resistance rates exceeded 30\% except Citrobacter spp. (28.6\%). Resistance rate to nitrofurantoin approached 100\% for all but Citrobacter 
Table 1 Cumulative resistance rates (2011-2019) of seven uropathogens to different antimicrobial agents

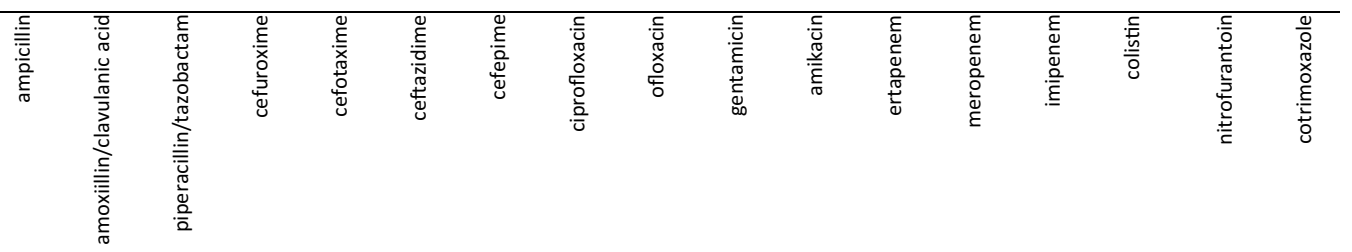

\begin{tabular}{|c|c|c|c|c|c|c|c|c|c|c|c|c|c|c|c|c|c|}
\hline Acinetobacter spp. & $94.5 \%$ & $93.0 \%$ & $16.7 \%$ & $90.5 \%$ & $50.7 \%$ & $30.6 \%$ & $22.9 \%$ & $30.6 \%$ & $17.7 \%$ & $12.5 \%$ & $11.1 \%$ & $36.1 \%$ & $5.6 \%$ & $5.6 \%$ & $33.3 \%$ & $91.2 \%$ & $25.7 \%$ \\
\hline Citrobacter spp. & $86.2 \%$ & $49.1 \%$ & $6.3 \%$ & $50.0 \%$ & $5.3 \%$ & $5.2 \%$ & $11.8 \%$ & $23.5 \%$ & $15.4 \%$ & $6.8 \%$ & $5.9 \%$ & $6.3 \%$ & $0.0 \%$ & $5.9 \%$ & $28.6 \%$ & $38.6 \%$ & $10.2 \%$ \\
\hline Enterobacter spp. & $96.2 \%$ & $88.6 \%$ & $27.9 \%$ & $93.6 \%$ & $20.2 \%$ & $15.3 \%$ & $18.8 \%$ & $31.5 \%$ & $12.4 \%$ & $17.0 \%$ & $1.8 \%$ & $10.3 \%$ & $1.8 \%$ & $1.8 \%$ & $34.5 \%$ & $59.1 \%$ & $22.3 \%$ \\
\hline Morganella morganii & $95.7 \%$ & $92.9 \%$ & $13.6 \%$ & $89.3 \%$ & $16.3 \%$ & $13.7 \%$ & $14.3 \%$ & $34.4 \%$ & $26.1 \%$ & $12.4 \%$ & $4.3 \%$ & $13.2 \%$ & $1.1 \%$ & $1.1 \%$ & $76.5 \%$ & $95.9 \%$ & $27.5 \%$ \\
\hline Providencia spp. & $91.7 \%$ & $87.2 \%$ & $8.0 \%$ & $55.3 \%$ & $19.1 \%$ & $15.2 \%$ & $3.8 \%$ & $37.0 \%$ & $35.9 \%$ & $33.3 \%$ & $0.0 \%$ & $0.0 \%$ & $0.0 \%$ & $0.0 \%$ & $50.0 \%$ & $94.3 \%$ & $33.3 \%$ \\
\hline Serratia spp. & $93.5 \%$ & $89.0 \%$ & $15.4 \%$ & $88.3 \%$ & $18.9 \%$ & $8.0 \%$ & $2.6 \%$ & $24.4 \%$ & $25.4 \%$ & $6.5 \%$ & $7.5 \%$ & $10.5 \%$ & $0.0 \%$ & $0.0 \%$ & $80.0 \%$ & $88.1 \%$ & $15.6 \%$ \\
\hline $\begin{array}{l}\text { Stenotrophomonas } \\
\text { maltophilia }\end{array}$ & $91.3 \%$ & $86.4 \%$ & $72.2 \%$ & $95.5 \%$ & $73.9 \%$ & $66.7 \%$ & $75.0 \%$ & $57.9 \%$ & $55.6 \%$ & $27.3 \%$ & $23.5 \%$ & $63.2 \%$ & $63.2 \%$ & $65.0 \%$ & $50.0 \%$ & $100.0 \%$ & $13.0 \%$ \\
\hline
\end{tabular}

Numbers refer to the percentage of resistant isolates of a pathogen to a particular antibiotic during the study period. Colour coding: green $=$ resistance rate $<20 \%$; yellow $=$ resistance rate $20-50 \%$; red $=$ resistance rate $>50 \%$

spp. (38.6\%). Cotrimoxazole resistance rates fluctuated between 10 and $30 \%$. Cotrimoxazole was, however, the antibiotic $S$. maltophilia had the highest susceptibility to (Additional file 1: Table S6).

\section{Discussion}

The uropathogens described in this report taken all together represent $5 \%$ of all positive urinary cultures from our urology department during the nine-year study period. Their low incidence likely explains the paucity of data in the literature on their prevalence and resistance patterns compared to other Gram-negative causative agents of UTIs. Some authors report resistance patterns for groups of these bacteria only [1, 26]; others focus on a single species within a genus $[27,28]$. This is where discrepancies between our and other authors' resistance data may stem from, apart from true differences in antimicrobial resistance observed across the world [6-15]. General informaion on the microorganisms covered in this report that is relevant to the topic is summarised in Table 2 .

In the present study, Acinetobacter resistance rate exceeded $30 \%$ for most antibiotics including ertapenem. It retained $>90 \%$ susceptibility to meropenem and imipenem and its resistance rates to piperacillin/tazobactam, ofloxacin and both aminoglycosides were within the clinically useful range of $<20 \%$ (Table 1 ). Thus, our Acinetobacter isolates were much more susceptible to most antimicrobials (except colistin) in comparison to a Spanish study from 2018 [29].

There is a limited amount of data on antimicrobial susceptibility of Citrobacter in clinical urinary isolates; Jiménez-Guerra et al. report a 10-year resistance patterns in urine samples $(n=65)$ from a tertiary Spanish hospital. Their resistance rates oscillate in line with ours except our three-fold and 5.5-fold higher resistance rate to cefepime and nitrofurantoin, respectively [28]. Of note, solely $C$. freundii was included in the Spanish study which may account for this difference. Fajfr et al. report on Citrobacter spp. $(n=97)$ resistance rates for ampicillin, ciprofloxacin and cotrimoxazole similar to our data [27].

Enterobacter, the most frequent pathogen in our study, retained extremely good susceptibility (>90\%) to amikacin, meropenem and imipenem only; clinically useful susceptibility rates were seen for ceftazidime, cefepime, ofloxacin, aminoglycosides and ertapenem. Cefepime resistance has shown a decreasing trend but this may be related to small numbers of Enterobacter isolates in the first half of the study period (Additional file 1: Table S2). A study from Asia-Pacific region reported similar resistance rates for cefepime and ertapenem; other antibiotics had different resistance profiles [15]. In comparison to a geographically closer Jiménez-Guerra et al. report [28], our Enterobacter strains were less resistant to imipenem (1.8\% vs. $8 \%)$ and more resistant to ciprofloxacin (31\% vs. $19 \%$ ). The inclusion of only a particular species 
Table 2 Summary of clinically relevant characteristics of the microorganisms covered in this work

\begin{abstract}
Acinetobacter spp. Originally presumed to be of little pathogenicity, Acinetobacter has emerged as a troublesome etiological agent of hospitalacquired infections worldwide. This strictly aerobic Gram-negative coccobacillus is able to accumulate diverse mechanisms of resistance leading to phenotypes resistant to most commercially available antibiotics. The mechanisms implicated in Acintetobacter spp. resistance include (1) decreased expression of bacterial porins, hindering the passage of beta-lactams into the periplasmic space (where they can attach to penicillin-binding proteins); (2) over-expression of bacterial efflux pumps, causing decreased concentration of beta-lactams (as well as quinolones and other antibiotics) in the periplasmic space; (3) mutations in gyrA and parC genes causing resistance to fluoroquinolones; (4) expression of aminoglycoside-modifying enzymes leading to resistance to this class of antibiotics; (5) chromosomally encoded inducible AmpC beta-lactamases conferring resistance to cephalosporins. Finally, Acinetobacter spp. acquired (plasmid-mediated) serine- and metallo-beta-lactamases confer resistance to carbapenems [23, 32].
\end{abstract}

Nosocomial UTIs are less frequent (most common Acinetobacter-related infection being ventilator-associated pneumonia) and are commonly diagnosed in elderly patients in ICU's, mostly men with indwelling catheters [23]. Acinetobacter survives for long periods on wet and dry surfaces and several studies have documented extensive contamination of the environment in the vicinity of colonised patients: bed linen, bed curtains, sink traps and hospital floor [23].

Citrobacter spp. Citrobacter, Enterobacter and Serratia are facultative anaerobic non-spore forming Gram-negative rods sometimes referred to as "CES" group and described together, due to the traits they share: (1) their biochemical characteristics; (2) prevalence and resistance patterns (observation corroborated by our data); (3) various intrinsic and acquired resistance mechanisms; and (4) the fact of being frequently associated with complicated UTIs, disease recurrence and prolonged treatment. Because of their AmpC betalactamase production, some authors group them with Pseudomonas spp. and indole-positive Proteus spp. to complete so-called "SPICE" group [33].

Citrobacter spp. is also one of the microorganisms implicated in the "purple urine bag syndrome", a conspicuous phenomenon of dubious clinical significance [34].

Enterobacter spp. The genus Enterobacter comprises of 22 species and is considered an opportunistic pathogen. While its pathogenicity and virulence remain rather unclear, its resistance mechanisms have been extensively studied. The production of beta-lactamase is a major mechanism of resistance to beta-lactams; E. aerogenes expresses AmpC beta-lactamase (cephalosporinase) that confers resistance to 1st generation cephalosporins but is inducible during treatment with a 3rd generation cephalosporin, leading again to resistance. Aminoglycoside-modifying enzymes are responsible for resistance to aminoglycosides and a mutation in one of their genetic determinants $\left(a a c-6^{\prime}-1 b\right)$ leads to a fluoroquinolone-resistant phenotype. A change in porin expression in the presence of imipenem leads to a decreased penetration of beta-lactams into E. aerogenes and bacterial efflux pumps remove fluoroquinolones and tetracyclines from Enterobacter spp. isolates [35]. Enterobacter is an opportunistic nosocomial pathogen: in a report from Taiwan, E. cloacae hospital outbreak has been described, attributale to a contaminated ureteroscope [36].

Serratia spp.

Originally presumed a non-pathogenic microorganism and even used a tracer in medical experiments, Serratia has established itself as an accepted clinical pathogen with widely prevalent multi-antibiotic resistant strains [37]. In urology wards, nosocomial outbreaks of $S$. liquefaciens and $S$. marcescens have been described related to cystometry appliance and urine bottles, respectively $[37,38]$. Indeed, urine-measurement containers, urinometers, urine-collecting basins and urinals as well as cystoscopy suite have been found to be reservoirs of $S$. marcescens [37].

Morganella spp. and Providencia spp.

Morganella and Providencia are sometimes (together with Proteus) grouped under the tribe Proteae. Originally not considered frank pathogens, they have emerged as importang causative agents of hospital-acquired infections in different organ systems [39]. Their common characteristics include (1) strong urease production; (2) frequent association with complicated UTIs, prolonged treatment and disease recurrence; (3) intrinsic resistance to nitrofurantoin and colistin (as confirmed by our data) and to tetracycline; (4) intrinsically decreased susceptibility to imipenem (not observed on our data); and (5) production of various beta-lactamases including AmpC [26].

Providencia frequently colonizes indwelling catheters; in a case-series $(n=14)$ of Providencia bacteraemia, UTI was identified as the source in $36 \%$ of cases and $71 \%$ of patients had an indwelling urinary catheter [40].

M. morganii, P. stuartii and P. rettgeri are among the causative microorganisms of the purple-urine bag syndrome (see above).

S. maltophilia

S. maltophilia intrinsic resistance mechanisms (low membrane permeability, chromosomally encoded multidrug resistance efflux pumps, beta-lactamases, antibiotic-modifying enzymes etc.) were suggested to have been acquired in non-human natural environments and not being due solely to clinical use of antibiotics. Its survival is facilitated by wet surfaces and aqueous solutions with minimal nutrients (e.g. drinking water, treated water, dialysate effluent) [24].

(E. cloacae) in the Spanish study may account for these differences.

Our Serratia spp. isolates exhibited highest resistance rates to ampicillin, amoxicillin/clavulanic acid, 2nd generation cephalosporins, colistin and nitrofurantoin, in line with its characteristics described in the literature [1]. Gajdács and Urbán [1] report on the cumulative resistance rates $(\mathrm{n}=1132$ in- and out-patients combined) of CES pathogens (Citrobacter, Enterobacter, Serratia) which are very close to our population $(n=392$ in- and out-patients combined): $28.8 \%$ versus $20.2 \%, 13.3 \%$ versus $17.0 \%$ and $19.1 \%$ versus $18.0 \%$, for ciprofloxacin, gentamicin and cotrimoxazole, respectively.

Providencia spp. had been presumed to be more resistant to antimicrobial drugs than Morganella spp. [26], but neither our nor others' data [26] support this general assumption. Providencia spp. retained very good susceptibility to piperacillin/tazobactam and cefepime and no resistant strains were isolated to amikacin and all carbapenems. No report on urinary isolates AMR rates for 
Providencia was found in the literature. For M. morganii, Jiménez-Guerra et al. report twice lower resistance rates to ciprofloxacin and cotrimoxazole and ten times higher resistance to imipenem (11\% vs. 1.1\%) [28] when compared to our data. M. morganii resistance rates for some of the relevant antibiotics (specifically cefotaxime, ceftazidime, amikacin and ertapenem) showed statistically significant increasing trends. Interestingly, resistance to cotrimoxazole was decreasing (Additional file 1: Table S2, S4-S6).

Although the majority of infections caused by $S$. maltophilia are respiratory tract infections $[24,30]-$ it is the least prevalent uropathogen in the present study-its significance as causative agent of UTIs should not be underestimated; not for its prevalence but more so for its far-reaching resistance. In our study, S. maltophilia resistance rate exceeded $50 \%$ for all antibiotics tested (including carbapenems); only aminoglycosides and cotrimoxazole showed more favourable resistance profiles. Its resistance to cotrimoxazole was in fact among the lowest in the present survey, despite a (statistically nonsignificant) increasing trend in our and others' data [31].

The limitations of the present report that need to be acknowledged include (1) the combination of both inand out-patients in the analysis; due to small numbers of isolates cultured from urinary samples, the authors did not find further division of the study population feasible (interested readers can find the division into in- and out-patients in Additional file 2: Table S7); (2) the impossibility to distinguish between community and hospitalacquired infections as the dates of urine cultures could not be linked to admission and discharge dates of each in-patient episode; (3) lack of identification of isolates down to species level. It might also be insightful to discriminate urine samples representing asymptomatic bacteriuria as opposed to a clinically symptomatic UTI but the nature of our retrospective data would not allow for this. Our report lacks information on fosfomycin due to the fact this agent has not been used in our health care system and therefore not tested in the microbiology laboratory.

To our knowledge, this report is one of the few in the literature systematically describing prevalence and resistance patterns of uropathogens covered herein. Similarity of our resistance data to those originating from not-sodistant regions (in instances where a direct comparison was possible) suggests that our data are applicable in a wider geographic area and not just in our own institution.

Monitoring of antibiotic resistance patterns is an important contribution to (1) providing a better service to patients by the likely best choice of antimicrobial empirical treatment; (2) economically rational allocation of resources in healthcare; and (3) the prevention of the global rapid spread of antibiotic resistance, a phenomenon that jeopardises many advances in medicine and surgery achieved over the last century. The knowledge of most efficiently acting antimicrobial agents (piperacilin/ tazobactam, carbapenems) should however not lead to their indiscriminate use as empirical treatment-perhaps with the exception of the gravest clinical scenarios.

\section{Conclusions}

The uropathogens described in this report are important microorganisms not for their prevalence but for their high resistance rates to a majority of commonly used antibiotics. Penicillin derivatives have generally poor effect except piperacillin/tazobactam. Cefuroxime is not efficient unlike cefotaxime, ceftazidime and cefepime (except against Acinetobacter spp. and S. maltophilia). Susceptibility to fluoroquinolones is limited. Amikacin is somewhat more efficient than gentamicine but susceptibilities for both safely exceed $80 \%$. Nitrofurantoin shows virtually no efficiency. Cotrimoxazole acts well against Citrobacter spp., Serratia spp. and it is the treatment of choice for S. maltophilia UTIs. Among carbapenems, ertapenem was less efficient than meropenem and imipenem except for S. maltophilia whose isolates were mostly not suceptible to any carbapenems.

\section{Abbreviations \\ AMR: Antimicrobial resistance; CES: Citrobacter, Enterobacter, Serratia; EUCAST: The European Committee on Antimicrobial Susceptibility Testing; ICU: Intensive care unit; MIC: Minimal inhibitory concentration; SPICE: Serratia, Pseudomonas, indole-positive Proteus, Citrobacter, Enterobacter; UTI: Urinary tract infection}

\section{Supplementary Information}

The online version contains supplementary material available at https://doi. org/10.1186/s12894-021-00821-8.

Additional file 1. Tables S1-S6: Yearly antimicrobial resistance rates of uropathogens to different antibiotics.

Additional file 2. Table S7: Yearly numbers of isolates for each uropathogen in the in-patient and out-patient population.

\section{Acknowledgements}

Not applicable.

\section{Authors' contributions}

$\mathrm{JH}$ : Conceptualization, Formal Analysis, Funding acquisition, Investigation, Methodology, Project administration, Visualization, Writing —original draft, Writing — review and editing; PC: Resources, Validation, Writing —review and editing; RZ: Data curation, Supervision, Writing —-review and editing. All authors read and approved the final manuscript.

\section{Funding}

Supported by MH CZ_DRO (Thomayer University Hospital_-TUH, 00064190). The funding body played no role in the analysis or interpretation of the data. 


\section{Availability of data and materials}

The anonymised raw datasets used and/or analysed during the current study are available from the corresponding author on reasonable request.

\section{Declarations}

\section{Ethics approval and consent to participate}

This is a retrospective analysis of anonymised data; therefore, an approval of the hospital ethical committee was not required (as per national legislation, Drugs and Medications Act no. 378/2007). All data processing was in compliance with the Declaration of Helsinki.

\section{Consent for publication}

Not applicable.

\section{Competing interests}

The authors declare that they have no competing interests.

\section{Author details}

1 Department of Urology, 3rd Faculty of Medicine, Charles University and Thomayer University Hospital, Videnska 800, 14059 Prague, Czech Republic. ${ }^{2}$ Department of Clinical Microbiology, Thomayer University Hospital, Videnska 800, Prague 14059, Czech Republic.

\section{Received: 28 September 2020 Accepted: 24 March 2021} Published online: 13 April 2021

\section{References}

1. Gajdács M, Urbán E. Resistance trends and epidemiology of CitrobacterEnterobacter-Serratia in urinary tract infections of inpatients and outpatients (RECESUTI): a 10-year survey. Medicina (B Aires). 2019;55(285):1-13.

2. Zowawi HM, Harris PNA, Roberts MJ, Tambyah PA, Schembri M, Pezzani $M D$, et al. The emerging threat of multidrug-resistant Gram-negative bacteria in urology. Nat Rev Urol. 2015;12:570-80. https://doi.org/10.1038/ nrurol.2015.199.

3. Schaeffer A, Schaeffer E. Infections of the urinary tract. In: Wein A, Kavoussi L, editors. Campbell-Walsh Urology. 10th ed. 2012. p. 258-60.

4. François M, Hanslik T, Dervaux B, Le Strat Y, Coignard B, Souty C, et al. The economic burden of urinary tract infections in women visiting general practices in France: a cross-sectional survey. BMC Health Serv Res. 2016;16(1):1-10. https://doi.org/10.1186/s12913-016-1620-2.

5. Vallejo-Torres L, Pujol M, Shaw E, Wiegand I, Vigo JM, Stoddart M, et al. Cost of hospitalised patients due to complicated urinary tract infections: a retrospective observational study in countries with high prevalence of multidrug-resistant Gram-negative bacteria: The COMBACTE-MAGNET, RESCUING study. BMJ Open. 2018;8(4):1-9.

6. Bonkat G, Bartoletti R, Bruyère F, Cai T, S.E. G, Köves B, et al. Urological infections [Internet]. 2020 [cited 2020 Sep 17]. https://uroweb.org/guide line/urological-infections/.

7. Cullen IM, Manecksha RP, Mccullagh E, Ahmad S, Kelly FO, Flynn RJ, et al. The changing pattern of antimicrobial resistance within 42033 Escherichia coli isolates from nosocomial, community and urology patient-specific urinary tract infections, Dublin, 1999-2009. BJUI Int. 2011;109(109):1198-206.

8. lacchini S, Sabbatucci M, Gagliotti C, Rossolini GM, Moro ML, lannazzo S, et al. Bloodstream infections due to carbapenemas eproducing Enterobacteriaceae in Italy: results from nationwide surveillance, 2014 to 2017. Eurosurveillance. 2019;24(5):66.

9. Lob SH, Nicolle LE, Hoban DJ, Kazmierczak KM, Badal RE, Sahm DF. Susceptibility patterns and ESBL rates of Escherichia coli from urinary tract infections in Canada and the United States, SMART 2010-2014. Diagn Microbiol Infect Dis. 2016:85(4):459-65

10. Guyomard-Rabenirina S, Malespine J, Ducat C, Sadikalay S, Falord M, Harrois $D$, et al. Temporal trends and risks factors for antimicrobial resistant Enterobacteriaceae urinary isolates from outpatients in Guadeloupe. BMC Microbiol. 2016:16:121. https://doi.org/10.1186/s12866-016-0749-9.
11. Sierra-Díaz E, Hernández-ríos CJ, Bravo-cuellar A. Antibiotic resistance: microbiological profile of urinary tract infections in Mexico. Circ Circ. 2019:87:176-82

12. Okeke IN, Laxminarayan R, Bhutta ZA, Duse AG, Jenkins P, Brien TFO, et al. AMR Resistance in developing countries. Lancet Infect Dis. 2005;5(August):481-93.

13. Fasugba O, Mitchell BG, Mnatzaganian G, Das A, Collignon P, Gardner A Five-year antimicrobial resistance patterns of urinary Escherichia coli at an Australian Tertiary Hospital: time series analyses of prevalence data. PLoS ONE. 2016;11(10):1-14

14. Hyun M, Noh Cl, Ryu SY, Kim HA. Changing trends in clinical characteristics and antibiotic susceptibility of Klebsiella pneumoniae bacteremia. Korean J Intern Med. 2018;33:595-603.

15. Karlowsky JA, Hoban DJ, Hackel MA, Lob SH, Sahm DF. Antimicrobial susceptibility of Gram-negative ESKAPE pathogens isolated from hospitalized patients with intra-abdominal and urinary tract infections in AsiaPacific countries: SMART 2013-2015. J Med Microbiol. 2017;66(1):61-9.

16. Klein EY, Van Boeckel TP, Martinez EM, Pant S, Gandra S, Levin SA, et al. Global increase and geographic convergence in antibiotic consumption between 2000 and 2015. Proc Natl Acad Sci USA. 2018:115(15):E3463-70.

17. Stapleton PJ, Lundon DJ, McWade R, Scanlon N, Hannan MM, O'Kelly F, et al. Antibiotic resistance patterns of Escherichia coli urinary isolates and comparison with antibiotic consumption data over 10 years, 2005-2014. Ir J Med Sci. 2017;186(3):733-41.

18. Kandil $\mathrm{H}$, Cramp E, Vaghela T. Trends in antibiotic resistance in urologic practice. Eur Urol Focus. 2016;2(4):363-73. https://doi.org/10.1016/j.euf. 2016.09.006.

19. Fleming-Dutra $K$, Hersh AL, Shapiro DJ. Prevalence of inappropriate antibiotic prescriptions among US ambulatory care visits, 2010-2011. JAMA 2016;315(17):1865-73.

20. Chardavoyne PC, Kasmire KE. Appropriateness of Antibiotic Prescriptions for urinary tract infections. West J Emerg Med. 2020;21(3):633-9.

21. Harris PNA, Wei JY, Shen AW, Abdile AA, Paynter S, Huxley RR, et al. Carbapenems versus alternative antibiotics for the treatment of bloodstream infections caused by Enterobacter, Citrobacter or Serratia species: a systematic review with meta-analysis. J Antimicrob Chemother. 2016;71(2):296-306

22. Thaden JT, Pogue JM, Kaye KS. Role of newer and re-emerging older agents in the treatment of infections caused by carbapenem-resistant Enterobacteriaceae. Virulence. 2017;8(4):403-16. https://doi.org/10.1080/ 21505594.2016.1207834.

23. Bergogne-Bérézin E, Towner KJ. Acinetobacter spp as nosocomial pathogens: microbiological, clinical, and epidemiological features. Clin Microbiol Rev. 1996;9(2):148-65.

24. Brooke JS. Stenotrophomonas maltophilia: an emerging global opportunistic pathogen. Clin Microbiol Rev. 2012;25(1):2-41.

25. Hrbacek J, Cermak P, Zachoval R. Current antibiotic resistance trends of uropathogens in central europe: survey from a tertiary hospital urology department 2011-2019. Antibiotics. 2020:9(9):1-11.

26. Gajdács M, Urbán E. Comparative epidemiology and resistance trends of proteae in urinary tract infections of inpatients and outpatients: a 10-year retrospective study. Antibiotics. 2019;8(91):1-13.

27. Fajfr M, Louda M, Paterová P, Ryšková L, Pacovský J, Košina J, et al. The susceptibility to fosfomycin of Gram-negative bacteria isolates from urinary tract infection in the Czech Republic: data from a unicentric study. BMC Urol. 2017;17(1):1-6.

28. Jiménez-Guerra G, Borrego-Jiménez J, Gutiérrez-Soto B, Expósito-Ruiz M, Navarro-Marí JM, Gutiérrez-F, et al. Evolución de la sensibilidad a los antibióticos de Enterobacter cloacae, Morganella morganii, Klebsiella aerogenes y Citrobacter freundii causantes de infecciones del tracto urinario: un estudio de vigilancia epidemiológica de 11 años. Enferm Infecc Microbiol Clin. 2019. https://doi.org/10.1016/j.eimc.2019.07.010.

29. Jiménez-Guerra G, Heras-Cañas V, Gutiérrez-Soto M, Aznarte-Padial M del P, Expósito-Ruiz M, Navarro-Marí JM, et al. Urinary tract infection by acinetobacter baumannii and pseudomonas aeruginosa: evolution of antimicrobial resistance and therapeutic alternatives. J Med Microbiol. 2018;67(6):790-7.

30. de Lemos MC, de Paixao EF, de Souza Santos MAR. Stenotrophomonas maltophilia isolates in a tertiary care hospital in Northeastern Brazil. Braz J Hea Rev. 2019;2(4):3373-84 
31. Nyc O, Matejková J. Stenotrophomonas maltophilia: significant contemporary hospital pathogen—review. Folia Microbiol Praha. 2010;55(3):286-94.

32. Munoz-Price $S$, Weinstein RA. Acinetobacter infection. N Engl J Med. 2008;358(12):1271-81.

33. Moy S, Sharma R. Treatment outcomes in infections caused by "SPICE" (Serratia, Pseudomonas, Indole-positive Proteus, Citrobacter, and Enterobacter) organisms: Carbapenem versus Noncarbapenem Regimens. Clin Ther. 2017;39(1):170-6.

34. Chassin-Trubert CA-M. Síndrome de la bolsa de orina púrpura: un fenómeno inusual y muy llamativo. Rev Med Chile. 2014;142:1482-4.

35. Davin-Regli A, Pagès J. Enterobacter aerogenes and Enterobacter cloacae; versatile bacterial pathogens confronting antibiotic treatment. Front Microbiol. 2015;6:392.

36. Chang C-L, Su L-H, Lu C-M, Tai F-T, Huang Y-C, Chang K-K. Outbreak of ertapenem-resistant Enterobacter cloacae urinary tract infections due to a contaminated ureteroscope. J Hosp Infect. 2013;85(2):118-24.
37. Mahlen SD. Serratia infections: from military experiments to current practice. Clin Microbiol Rev. 2011;24(4):755-91.

38. Su L, Ou JT, Leu H, Chiang P, Chiu Y, Chia J, et al. Extended epidemic of nosocomial urinary tract infections caused by Serratia marcescens. J Clin Microbiol. 2003;41(10):4726-32.

39. O'Hara CM, Brenner FW, Miller JM. Classification, identification, and clinical significance of Proteus, Providencia, and Morganella. Clin Microbiol Rev. 2000;13(4):534-46

40. Choi HK, Kim YK, Kim HY, Park JE, Uh Y. Clinical and microbiological features of Providencia bacteremia: experience at a tertiary care hospital. Korean J Intern Med. 2015;30:219-25.

\section{Publisher's Note}

Springer Nature remains neutral with regard to jurisdictional claims in published maps and institutional affiliations.
Ready to submit your research? Choose BMC and benefit from:

- fast, convenient online submission

- thorough peer review by experienced researchers in your field

- rapid publication on acceptance

- support for research data, including large and complex data types

- gold Open Access which fosters wider collaboration and increased citations

- maximum visibility for your research: over $100 \mathrm{M}$ website views per year

At BMC, research is always in progress.

Learn more biomedcentral.com/submissions 